\title{
Development and Validation of the Tele-Pulmonary Rehabilitation Acceptance Scale
}

\author{
Abdullah A Almojaibel, Niki Munk, Lynda T Goodfellow, Thomas F Fisher, Kristine K Miller, \\ Amber R Comer, Tamilyn Bakas, and Michael D Justiss
}

\begin{abstract}
BACKGROUND: Using telehealth in pulmonary rehabilitation (telerehabilitation) is a new field of health-care practice. To successfully implement a telerehabilitation program, measures of acceptance of this new type of program need to be assessed among potential users. The purpose of this study was to develop a scale to measure acceptance of using telerehabilitation by health-care practitioners and patients. METHODS: Three objectives were met (a) constructing a modified scale of the technology acceptance model, (b) judging the items for content validity, and (c) judging the scale for face validity. Nine experts agreed to participate and evaluate item relevance to theoretical definitions of domains. To establish face validity, 7 health-care practitioners and 5 patients were interviewed to provide feedback about the scale's clarity and ease of reading. RESULTS: The final items were divided into 2 scales that reflected the health-care practitioner and patient responses. Each scale included 3 subscales: perceived usefulness, perceived ease of use, and behavioral intention. CONCLUSIONS: The 2 scales, each with 3 subscales, exhibited evidence of content validity and face validity. The 17-item telerehabilitation acceptance scale for health-care practitioners and the 13-item telerehabilitation acceptance scale among patients warrant further psychometric testing as valuable measures for pulmonary rehabilitation programs. Key words: telehealth; telerehabilitation; acceptance; pulmonary rehabilitation; chronic respiratory diseases; health-care practitioners; content validity; face validity. [Respir Care 2019;64(9):1057-1064. (C) 2019 Daedalus Enterprises]
\end{abstract}

\section{Introduction}

Chronic pulmonary diseases are among the most challenging for health-care providers to manage because of the

\footnotetext{
Dr Almojaibel is affiliated with the Department of Respiratory Care, College of Applied Medical Sciences, University of Imam Abdulrahman bin Faisal, Dammam, Saudi Arabia. Dr Munk is affiliated with the Department of Health Sciences, School of Health and Rehabilitation Sciences, Indiana University, School of Health and Rehabilitation Sciences, Indianapolis, Indiana. Dr Goodfellow is affiliated with Lewis College of Nursing and Health Professions, Georgia State University, Atlanta, Georgia. Dr Fisher is affiliated with Dwyer College of Health Sciences, IU South Bend, South Bend, Indiana. Dr Miller is affiliated with the Physical Therapy Program, School of Health and Rehabilitation Sciences, Indiana University, Indianapolis, Indiana. Dr Comer is affiliated with Health Sciences, Indiana University, Indianapolis, Indiana. Dr Bakas PhD is affiliated with the University of Cincinnati College of Nursing, Cincinnati, Ohio. Dr Justiss is affiliated with the Department of Occupational Therapy, School of Applied Health Sciences, Brooks Rehabilitation College of Healthcare Sciences, Jacksonville University, Jacksonville, Florida.
}

The authors have disclosed no conflicts of interest. wide-ranging impacts on patients' medical, social, and economic status. ${ }^{1}$ The treatment plan for patients with chronic pulmonary diseases should improve their well-being and functional status. Pulmonary rehabilitation can be used as a facilitator for coping with the long-term effects of chronic respiratory disease, even after maximizing medical management. ${ }^{2}$ The American Thoracic Society and the European Respiratory Society define pulmonary rehabilitation as the following: A comprehensive intervention based on a thorough patient assessment followed by patient-tailored therapies stretching across, education exercise training, and

\footnotetext{
Supplementary material related to this paper is available at http:// www.rcjournal.com.

Correspondence: Abdullah A Almojaibel PhD RRT, Department of Respiratory Care, College of Applied Medical Sciences, University of Imam Abdulrahman bin Faisal, King Faisal Road, Alrakkah, PO Box 2435, Dammam, Saudi Arabia 31441. E-mail: amojaibel@iau.edu.sa.
}

DOI: $10.4187 /$ respcare. 06432 
behavior change, designed to improve the physical and psychological condition of people suffering from chronic respiratory disease and to promote the long-term adherence to health-enhancing behaviors. ${ }^{3}$

Providing pulmonary rehabilitation services in the current health-care system is challenging due to the increasing number of patients with chronic respiratory diseases and the apparent shortage in health-care providers and pulmonary rehabilitation programs. ${ }^{4}$ As found by Alsubaiei et al, ${ }^{5}$ a lack of hospital capacity, trained health-care providers, and funds were barriers for setting up pulmonary rehabilitation programs in Saudi Arabia. Further complicating this challenge is underutilization of pulmonary rehabilitation programs where they are available. ${ }^{6}$ Keating et $\mathrm{al}^{7}$ found that the percentage of nonattendance in pulmonary rehabilitation ranged from 8.3 to $49.6 \%$, and noncompleters ranged from 9.7 to $31.8 \%$. Low utilization rates were associated with poor access to a rehabilitation program, lack of transportation, and inconvenient timing of the programs for patients. Telehealth and telerehabilitation are emerging modes of providing health-care services, with the potential to address these issues and improve patients' pulmonary rehabilitation program participation and adherence.

Telehealth is the use of telecommunication technology and computers to provide and receive clinical health-care services. Application of telehealth in pulmonary rehabilitation (telerehabilitation) is a new and promising field of health-care practice. As a new field of practice, it is important to understand the potential barriers to implementation of telerehabilitation in pulmonary rehabilitation, such as uncertainty and misperceptions held by future users of the technology. ${ }^{8}$ Specifically, determinants of acceptance to use telerehabilitation need to be identified among potential users, such as health-care providers and patients, before implementation. The influence of these factors has not been previously studied or reported, even though it is vitally important to support the development of telerehabilitation systems that consider patients' and health providers' needs. ${ }^{9}$

Increased interest in telehealth activities has led to more focus on understanding users' intentions to use telehealth. Users' acceptance of telehealth is suggested as one of the determinants of future use and adherence to telehealth services. ${ }^{10}$ Specifically, health professionals' acceptance of telehealth has been identified as a key factor that affects success and sustainability of telehealth programs, ${ }^{4}$ and lack of staff acceptance of telehealth has been reported as a potential barrier to telehealth implementation. ${ }^{8}$ Nonacceptance among potential telehealth users may lead to low levels of utilization of the proposed telehealth program. ${ }^{11}$ Analysis of these data indicate that understanding the potential users' acceptance of telehealth will lead to successful, high-quality, and safe implementation of telehealth programs. ${ }^{12}$ Patients with low lev-

\section{QUICK LOOK}

\section{Current knowledge}

Telerehabilitation is a new method of providing rehabilitation services via the internet for patients at home. Understanding acceptance of using telerehabilitation is a key element in ensuring successful implementation. Currently, there is no scale with evidence of validity and reliability that could be used to measure acceptance of using telerehabilitation among potential users.

\section{What this paper contributes to our knowledge}

This study provided evidence of content and face validity of 2 newly developed scales that could be used to measure telerehabilitation acceptance among health-care practitioners and patients. The content and face validity of the scales were established by a group of experts in fields related to telerehabilitation and a group of potential users of telerehabilitation.

els of telehealth acceptance might use telehealth services less, which could reduce the potential benefits of the program. ${ }^{10}$ Understanding potential users' determinants of telerehabilitation acceptance could increase telerehabilitation program usage, ease the implementation of telerehabilitation, and decrease the gap between expectations of the telerehabilitation programs and the reality that may include difficulties. ${ }^{4}$ The purpose of this study was to develop a scale with evidence of validity to measure acceptance of using telerehabilitation from the perspectives of health-care practitioners and patients.

\section{Methods}

Development and validation of the Tele-Pulmonary Rehabilitation Acceptance Scale study was designed to meet 3 objectives: constructing a modified scale of the technology acceptance model, judging the items for content validity, and judging the scale for face validity.

\section{Model Construction}

The technology acceptance model is a theoretical model developed by Fred D. Davies (1985) ${ }^{13}$ to describe the key factors that contribute to information technology acceptance, which can help to discover the problems in any new system before implementation. According to the technology acceptance model, perceived usefulness and perceived ease of use are the major determinants of the positive intention to use technology (Fig. 1). ${ }^{13}$ The technology acceptance model is considered one of the most used among theoretical models that explain users' acceptance of tech- 


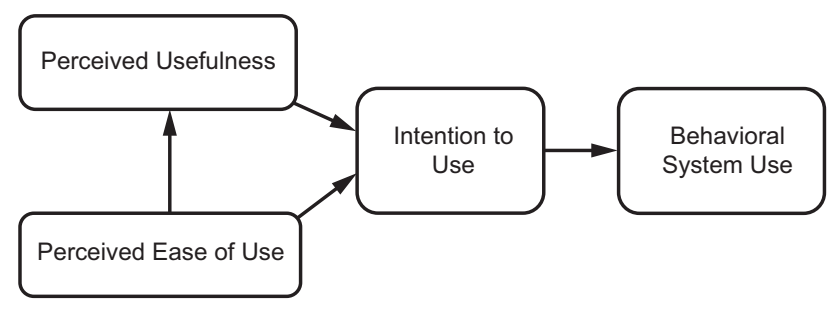

Fig. 1. The technology acceptance model.

nology. ${ }^{14}$ The technology acceptance model is very powerful in identifying factors that influence acceptance of computer technology. At the same time, using the technology acceptance model is considered easy and simple. ${ }^{15}$

Constructs of the Technology Acceptance Model. People tend to use new technology systems when they believe that the new technology will help them to perform better. This can be referred as perceived usefulness. Perceived usefulness is defined as "the degree to which a person believes that using a particular system would enhance his or her job performance." 13 People also consider whether the new system is hard to use, which speaks to the second influence of acceptance perceived ease of use. Perceived ease of use is defined as "the degree to which a person believes that using a particular system would be free of effort." ${ }^{13}$ Although multiple studies confirmed the validity and reliability of the technology acceptance model, ${ }^{16,17}$ additional studies are needed to validate the utilization of the technology acceptance model, especially in the context of telehealth and telerehabilitation.

\section{Content Validity}

Fifteen experts were invited to participate in the content validity assessment. Experts were required to have one of the following: a doctorate or master's degree in healthcare-related fields; papers published in the field of pulmonary rehabilitation, telehealth, information technology, or scale development; or experience with working in pulmonary rehabilitation or telehealth. The evaluation form used in this assessment consisted of relevance ratings for each item, with a column for experts to provide comments (see the supplementary materials at http://www.rcjournal. com). The content experts evaluated each item for wording, layout, clarity, redundancy, and relevance to the scale's domains based on the theoretical definitions of the domains. Two rounds of content validity assessments were conducted. The first round was to categorize the items and rate their relevance.

In round 1, the reviewers categorized each item as falling under the perceived usefulness or perceived ease of use domain. Then, the experts evaluated each item's relevance to the assigned domain by using a 4-point Likert scale: 1, not relevant; 2 , relevant, needs major revision; 3 , moderately relevant, needs minor revision; and 4 , very relevant, no modification. The reviewers also evaluated the relevance of the behavioral intention items by using the same 4-point Likert scale. In round 1 of the evaluation, the item content validity index was calculated for each item. The item content validity index is the number of reviewers who give a rating of either 3 or 4 for an item relevance (moderately relevant or very relevant) divided by the total number of reviewers. ${ }^{18}$ Round 2 of the evaluation was to finalize the inclusion of the items. In this evaluation, each item was followed by a dichotomous scale with 2 options for inclusion: yes or no. The scale content validity index was calculated for each subscale. The scale content validity index is the proportion of highrated items that received 3 or 4 in the 4-point relevance scale by the raters. ${ }^{18}$

\section{Face Validity}

In this study, face validity was the extent to which the scale reflected factors that affect acceptance of using telerehabilitation in the future. Seven health-care practitioners who worked in pulmonary rehabilitation programs participated in this assessment, including 1 physician, 3 nurses, 1 physiotherapist, and 3 respiratory therapists. The physiotherapist was working in Saudi Arabia and the rest of the participants were working at Indiana University (IU) Health Hospitals, Indiana. Five patients who attended traditional out-patient pulmonary rehabilitation programs agreed to participate in evaluating the survey questions. Four of the participants in this face validity assessment were attending the out-patient pulmonary rehabilitation center at IU Health Hospital, Indianapolis, Indiana. One of the participants was attending the out-patient pulmonary rehabilitation center at St Vincent Rehabilitation Center, Indianapolis, Indiana. During in-person interviews, telephone calls, or e-mails, each participant was provided with the final version of the Tele-Pulmonary Rehabilitation Acceptance Scale in both electronic and paper-based formats. To get participants' feedback, 3 openended questions were asked after completing the survey: How do you rate the scale's instruction and items in terms of clarity and ease of read, how do you rate the clarity of the demographic questions, and do you suggest additional questions for the demographic questions? This study was approved by the Indiana University institutional review boards. All the participants were informed about the purpose and methods of the study. They were informed that participation in this study was voluntary and that their responses would be confidential. This study was conducted at Indiana University Purdue University at Indianapolis, School of Health and Rehabilitation Sciences, Indianapolis, Indiana. 
Table 1. Items Categorization Based on Reviewers' Evaluation From Round 1

\begin{tabular}{|c|c|c|c|}
\hline $\begin{array}{l}\text { Item } \\
\text { No. }\end{array}$ & Items Pool & $\begin{array}{c}\text { Raters' } \\
\text { CVI }\end{array}$ & $\begin{array}{l}\text { Assigned } \\
\text { Category }\end{array}$ \\
\hline 1 & Telerehabilitation will allow me to do my tasks more quickly & 0.55 & PEOU \\
\hline 2 & Telerehabilitation will allow me to accomplish more than face-to-face rehabilitation & 0.67 & PU \\
\hline 3 & Telerehabilitation will give me greater control over my disease symptoms. & 0.89 & PU \\
\hline 4 & Telerehabilitation will save me time & 0.67 & PU \\
\hline 5 & Telerehabilitation will be flexible to use & 0.89 & PEOU \\
\hline 6 & Telerehabilitation will improve access to the rehabilitation programs & 0.89 & PU \\
\hline 7 & Learning to operate the telerehabilitation equipment will be easy for me & 1.00 & PEOU \\
\hline 8 & It will be easy to get the telerehabilitation equipment to do what I want it to do & 1.00 & PEOU \\
\hline 9 & My interaction with the telerehabilitation equipment will be clear & 0.78 & PEOU \\
\hline 10 & Telerehabilitation will be easy to use & 0.89 & PEOU \\
\hline 11 & $\begin{array}{l}\text { Providing and/or receiving pulmonary rehabilitation services by using telerehabilitation will be } \\
\text { more convenient }\end{array}$ & 0.56 & PU \\
\hline 12 & Using telerehabilitation technology will be understandable & 0.89 & PEOU \\
\hline 13 & Telerehabilitation will meet my needs & 0.78 & PU \\
\hline 14 & Using telerehabilitation will improve my performance & 0.78 & PU \\
\hline 15 & Telerehabilitation will increase the quality of the pulmonary rehabilitation services & 0.78 & PU \\
\hline 16 & Telerehabilitation will improve my attendance in the rehabilitation program & 0.78 & PU \\
\hline 17 & Telerehabilitation will cancel transportation difficulties in getting to the rehabilitation center & 0.67 & PU \\
\hline 18 & It will be easy for me to become skillful in using telerehabilitation equipment & 0.89 & PEOU \\
\hline 19 & Telerehabilitation will decrease the cost of the rehabilitation program & 0.67 & PU \\
\hline 20 & Using telerehabilitation will be simple & 1.00 & PEOU \\
\hline 21 & Telerehabilitation will facilitate monitoring of the disease & 1.00 & PU \\
\hline 22 & Telerehabilitation will give me the feeling of being safe & 0.78 & PU \\
\hline 23 & Telerehabilitation will improve the rehabilitation plan & 1.00 & PU \\
\hline 24 & Telerehabilitation will give me the feeling of being continuously monitored & 0.89 & PU \\
\hline 25 & Telerehabilitation could help me provide and/or receive care more quickly & 0.89 & PU \\
\hline 26 & Education sessions will be easier when using telerehabilitation & 0.78 & PEOU \\
\hline 27 & Telerehabilitation will be useful in the rehabilitation program & 1.00 & PU \\
\hline 28 & Telerehabilitation will save me time of travelling to the health care center & 0.56 & PU \\
\hline 29 & Telerehabilitation will improve the relationship between the health-care provider and the patient & 1.00 & PU \\
\hline 30 & Telerehabilitation does not require a lot of my mental effort & 0.89 & PEOU \\
\hline \multicolumn{4}{|c|}{$\begin{array}{l}\mathrm{CVI}=\text { content validity index } \\
\mathrm{PEOU}=\text { perceived ease of use } \\
\mathrm{PU}=\text { perceived usefulness }\end{array}$} \\
\hline
\end{tabular}

\section{Results}

\section{Content Validity Findings}

An evaluation form was created for the newly developed items for both health-care practitioners and patients. Of the 15 content experts, 9 agreed to participate and completed the evaluation form. Data from round 1 were categorized based on the reviewers' evaluation (Table 1). The item "content validity index" was calculated for each item (Tables 2 and 3). To construct the perceived usefulness and the perceived ease of use scales, items with content validity indexes of $\geq 0.83$ were directly included in the scale to be evaluated in round 2 . Of the 30 items in the perceived usefulness and perceived ease of use item pool, 14 items were rated with content validity indexes of $\geq 0.83$. Only 3 items from the perceived usefulness and perceived ease of use items pool with content validity indexes of 0.78 were included in the final items list (items 5, 11, and 20). From the behavioral intention (item pool, 3 items met the criteria of content validity indexes of $\geq 0.83$. One item was rated with a content validity index of 0.78 (behavioral intention 1).

The scale content validity indexes for the first evaluation round for each of the subscales (perceived usefulness and perceived ease of use) and (behavioral intention) were 0.84 and 0.80 , respectively. All items with content validity indexes of $\geq 0.83$ were used to create 2 lists of items (perceived usefulness or perceived ease of use) based on reviews' categorization. The experts suggested writing 2 scales; one intended to measure acceptance of using telerehabilitation among health-care practitioners and one intended to measure acceptance of using telerehabilitation 
Table 2. Perceived Usefulness and Perceived Ease of Use Items' CVIs and Scale Content Validity Index (S-CVI) From Round 1

\begin{tabular}{|c|c|c|}
\hline $\begin{array}{l}\text { Item } \\
\text { No. }\end{array}$ & Items Pool & CVI \\
\hline 1 & Telerehabilitation will allow me to do my tasks more quickly & 0.78 \\
\hline 2 & Telerehabilitation will allow me to accomplish more than face-to-face rehabilitation & 0.78 \\
\hline 3 & Telerehabilitation will give me greater control over my disease symptoms & 0.78 \\
\hline 4 & Telerehabilitation will save me time & 0.89 \\
\hline 5 & Telerehabilitation will be flexible to use* & 0.78 \\
\hline 6 & Telerehabilitation will improve access to the rehabilitation programs & 0.89 \\
\hline 7 & Learning to operate the telerehabilitation equipment will be easy for me & 1.00 \\
\hline 8 & It will be easy to get the telerehabilitation equipment to do what I want it to do & 0.67 \\
\hline 9 & My interaction with the telerehabilitation equipment will be clear & 0.67 \\
\hline 10 & Telerehabilitation will be easy to use & 1.00 \\
\hline 11 & Providing and/or receiving pulmonary rehabilitation services by using telerehabilitation will be more convenient* & 0.78 \\
\hline 12 & Using telerehabilitation technology will be understandable & 0.78 \\
\hline 13 & Telerehabilitation will meet my needs & 0.78 \\
\hline 14 & Using telerehabilitation will improve my performance & 0.78 \\
\hline 15 & Telerehabilitation will increase the quality of the pulmonary rehabilitation services & 0.78 \\
\hline 16 & Telerehabilitation will improve my attendance in the rehabilitation program & 1.00 \\
\hline 17 & Telerehabilitation will cancel transportation difficulties in getting to the rehabilitation center & 1.00 \\
\hline 18 & It will be easy for me to become skillful in using telerehabilitation equipment & 0.89 \\
\hline 19 & Telerehabilitation will decrease the cost of the rehabilitation program & 0.67 \\
\hline 20 & Using telerehabilitation will be simple* & 0.78 \\
\hline 21 & Telerehabilitation will facilitate monitoring of the disease & 0.89 \\
\hline 22 & Telerehabilitation will give me the feeling of being safe & 0.56 \\
\hline 23 & Telerehabilitation will improve the rehabilitation plan & 0.78 \\
\hline 24 & Telerehabilitation will give me the feeling of being continuously monitored & 1.00 \\
\hline 25 & Telerehabilitation could help me provide and/or receive care more quickly & 1.00 \\
\hline 26 & Education sessions will be easier when using telerehabilitation & 0.89 \\
\hline 27 & Telerehabilitation will be useful in the rehabilitation program & 0.89 \\
\hline 28 & Telerehabilitation will save me time of travelling to the health care center & 1.00 \\
\hline 29 & Telerehabilitation will improve the relationship between the health care provider and the patient & 0.89 \\
\hline 30 & Telerehabilitation does not require a lot of my mental effort & 0.67 \\
\hline \multicolumn{3}{|c|}{$\begin{array}{l}\text { * Items with CVI } \leq 0.78 \text { and included in the next evaluation after revision. } \\
\mathrm{CVI}=\text { content validity index } \\
\mathrm{S}-\mathrm{CVI}=\text { the proportion of items that achieved a rating of } 3 \text { or } 4 \text { by all the reviewers } \\
\text { S-CVI Ave }=\text { average of the I-CVIs }=0.84\end{array}$} \\
\hline
\end{tabular}

among patients with chronic respiratory diseases. Items retrieved from round 1 were listed in 2 scales to be reevaluated in round 2 .

In the round 2 evaluation, only 7 evaluation forms were completed and returned by the experts on the review panel. Items with content validity indexes of $\geq 0.78$ were included in the final version of the scales. Only one item with a content validity index of 0.71 was included in the patient version of the scale (item 10), and only one item with a content validity index of 0.71 (item 7) was included in the health-care practitioners version of the scale. The final items were divided into 2 scales. One scale was designed to measure telerehabilitation acceptance among patients with chronic respiratory diseases and the other was designed to measure telerehabilitation acceptance among health-care practitioners working in pulmonary rehabilitation programs (Tables 4 and 5).

\section{Face Validity Assessments}

Face validity assessment of the Tele-Pulmonary Rehabilitation Acceptance Scale was conducted by a group of healthcare practitioners and a group of patients. Seven health-care practitioners who worked in pulmonary rehabilitation programs participated in the face validity assessment. The patients' version of the Tele-Pulmonary Rehabilitation Acceptance Scale was reviewed by 5 patients who attended traditional pulmonary rehabilitation programs. All the items were deemed appropriate and clear based on the questions presented to the health-care practitioners and the patients during face validity evaluations.

\section{Discussion}

In tandem with the growth of telehealth, there is an increasing interest in the rehabilitation field in using 
telehealth and in switching to what is called telerehabilitation. ${ }^{19}$ Results of this scale-development study provided evidence of content and face validity of the TelePulmonary Rehabilitation Acceptance Scale. This study

Table 3. Behavioral Intention Items' CVIs and Scale Content Validity Index (S-CVI) From Round 1

\begin{tabular}{|c|c|c|}
\hline Item No. & Behavioral Intention Items Pool & CVI \\
\hline 1 & I am positive toward using the telerehabilitation* & 0.78 \\
\hline 2 & $\begin{array}{l}\text { I will use the telerehabilitation when it becomes } \\
\text { available }\end{array}$ & 0.89 \\
\hline 3 & $\begin{array}{l}\text { I am willing to use telerehabilitation to provide } \\
\text { or receive pulmonary rehabilitation services }\end{array}$ & 0.89 \\
\hline 4 & $\begin{array}{l}\text { I will use the telerehabilitation to provide or } \\
\text { receive pulmonary rehabilitation services as } \\
\text { often as needed }\end{array}$ & 1. \\
\hline 5 & $\begin{array}{l}\text { I will use the telerehabilitation to provide or } \\
\text { receive pulmonary rehabilitation services } \\
\text { rather than the traditional face-to-face } \\
\text { sessions }\end{array}$ & 0. \\
\hline 6 & I will usually use telerehabilitation & 0. \\
\hline
\end{tabular}

* Items with CVI $\leq 0.78$ and included in the next evaluation after revision. These items we exempted from the 0.83 criteria.

$\mathrm{CVI}=$ content validity index

$\mathrm{S}-\mathrm{CVI}=$ the proportion of items that achieved a rating of 3 or 4 by all the reviewers

$\mathrm{S}$-CVI Ave $=$ average of the I-CVIs $=0.80$ developed 2 versions of the Tele-Pulmonary Rehabilitation Acceptance Scale. One version with 13 items to measure telerehabilitation acceptance among patients with chronic respiratory diseases and one version with 17 items to measure telerehabilitation acceptance among health-care practitioners who worked in pulmonary rehabilitation programs. The scale developed herein can be used to collect data from health-care practitioners who worked in pulmonary rehabilitation programs and patients who attended pulmonary rehabilitation programs. Understanding potential users' intentions to use telerehabilitation is a key factor in ensuring successful and prolonged implementation. ${ }^{12}$

To our knowledge, this was the first study to develop and validate a psychometric instrument to measure telerehabilitation acceptance among health-care practitioners who worked in pulmonary rehabilitation programs and patients who attended pulmonary rehabilitation programs. One relevant study used the unified theory of acceptance and use of technology to examine factors that affect acceptance of new technologies for rehabilitation by therapists but did not report the process of content validity. ${ }^{20}$ The scope of telerehabilitation in this same study was different than the telerehabilitation practice of interest in our acceptance measurement, which included the use of telecommunication technologies to provide and receive rehabilitation services for

Table 4. Patients' Version of the Telerehabilitation Acceptance Scale

\begin{tabular}{|c|c|c|}
\hline Items & Item No. & I-CVI \\
\hline \multicolumn{3}{|l|}{ PU of telerehabilitation } \\
\hline Telerehabilitation will improve my access to rehabilitation programs & 6 & 1.00 \\
\hline Telerehabilitation will improve my attendance in the rehabilitation program & 16 & 0.86 \\
\hline Telerehabilitation will eliminate transportation difficulties in getting to the rehabilitation center & 17 & 1.00 \\
\hline Telerehabilitation could help me to receive care more quickly at home & 25 & 0.86 \\
\hline Telerehabilitation will be useful in the rehabilitation program & 27 & 1.00 \\
\hline Telerehabilitation will improve my communication with the health-care provider & 29 & 1.00 \\
\hline Telerehabilitation will improve my commitment to the rehabilitation program & 32 & NA \\
\hline Patients' PU scale-CVI: PEOU of telerehabilitation & & 0.82 \\
\hline Learning to operate the telerehabilitation equipment will be easy for me & 7 & 0.86 \\
\hline Telerehabilitation will be easy to use & 10 & 0.71 \\
\hline Receiving pulmonary rehabilitation services at home by using telerehabilitation will be more convenient & 11 & 0.86 \\
\hline Education sessions will be easier when using telerehabilitation & 26 & 1.00 \\
\hline Patients' PEOU scale-CVI: BI to use telerehabilitation & & 0.89 \\
\hline BI 3: I will plan to use telerehabilitation to receive pulmonary rehabilitation services & NA & 1.00 \\
\hline $\begin{array}{l}\text { BI 4: I will use telerehabilitation to receive pulmonary rehabilitation services as often as } \\
\text { recommended by my provider }\end{array}$ & NA & 0.86 \\
\hline Patients’ BI scale-CVI & NA & 0.93 \\
\hline Scale-CVI & NA & 0.88 \\
\hline \multicolumn{3}{|l|}{$\begin{array}{l}\text { I-CVI }=\text { item content validity index } \\
\text { PU = perceived usefulness } \\
\text { NA = not applicable } \\
\text { PEOU = perceived ease of use } \\
\text { CVI = content validity index } \\
\text { BI = behavioral intention }\end{array}$} \\
\hline
\end{tabular}


Table 5. Health-Care Practitioners' Version of the Telerehabilitation Acceptance Scale

\begin{tabular}{|c|c|c|}
\hline Items & Item No. & I-CVI \\
\hline \multicolumn{3}{|l|}{ PU of telerehabilitation } \\
\hline Telerehabilitation will save me time & 4 & 1.00 \\
\hline Telerehabilitation will improve patients' access to rehabilitation programs & 6 & 1.00 \\
\hline Telerehabilitation will improve patients' attendance in the rehabilitation program & 16 & 1.00 \\
\hline Telerehabilitation will facilitate monitoring of the patients' disease symptoms & 21 & 1.00 \\
\hline Telerehabilitation could help me to provide care more quickly for patients at home & 25 & 1.00 \\
\hline Telerehabilitation will be useful in the rehabilitation program & 27 & 1.00 \\
\hline Telerehabilitation will improve my communication with patients & 29 & 0.86 \\
\hline Telerehabilitation will facilitate monitoring of the patients' daily activities & 31 & 0.86 \\
\hline Telerehabilitation will improve patients' adherence to the rehabilitation program & 32 & NA \\
\hline \multicolumn{3}{|l|}{ Health-care practitioner' PU scale-CVI } \\
\hline PEOU of telerehabilitation & & 0.86 \\
\hline Learning to operate the telerehabilitation equipment will be easy for me & 7 & 0.71 \\
\hline Telerehabilitation will be easy to use & 10 & 1.00 \\
\hline Providing pulmonary rehabilitation services by using telerehabilitation will be more convenient & 11 & 0.86 \\
\hline Education sessions will be easier when using telerehabilitation & 26 & 1.00 \\
\hline \multicolumn{3}{|l|}{ Health-care practitioner' PEOU scale-CVI } \\
\hline BI to use telerehabilitation & NA & 0.89 \\
\hline BI 1: I feel positive about using telerehabilitation & NA & 0.86 \\
\hline BI 2: I will use telerehabilitation when it becomes available in my rehabilitation center & NA & 1.00 \\
\hline BI 3: I will use telerehabilitation to provide pulmonary rehabilitation services & NA & 1.00 \\
\hline $\begin{array}{l}\text { BI 4: I will use telerehabilitation to provide pulmonary rehabilitation services as often as } \\
\text { recommended by the care team }\end{array}$ & NA & 0.86 \\
\hline Health-care practitioner' BI scale-CVI & NA & 0.93 \\
\hline Scale-CVI & NA & 0.83 \\
\hline $\begin{array}{l}\text { I-CVI = item content validity index } \\
\text { PU = perceived usefulness } \\
\text { NA = not applicable } \\
\text { PEOU = perceived ease of use } \\
\text { CVI = content validity index } \\
\text { BI = behavioral intention } \\
\text { Scale-CVI = Scale Content Validity Index }\end{array}$ & & \\
\hline
\end{tabular}

patients at home. Another recent study explored the technology engagement level of people who attended pulmonary rehabilitation and its effect on their intention to use telerehabilitation. ${ }^{21}$ Even though the main goal of this second study was to only assess the level of technology engagement and its effect on the intention to use telerehabilitation, it is a key study that examined how the demographics of patients might influence their intentions to use telerehabilitation when it is available in their rehabilitation facility.

The goal of our study was to develop and validate a tool to measure acceptance of using telerehabilitation among the key potential users, including health-care practitioners and patients. Now that evidence of content validity and face validity of the Tele-Pulmonary Rehabilitation Acceptance Scale was obtained, further studies are warranted to measure telerehabilitation acceptance of health-care practitioners and patients. Further studies are also needed to provide evidence of internal consistency reliability and construct validity of the TelePulmonary Rehabilitation Acceptance Scale. The Tele-
Pulmonary Rehabilitation Acceptance Scale shows great potential as a useful measure to determine acceptance of using telerehabilitation among health-care practitioners and patients with chronic respiratory conditions.

\section{Limitations}

There were several limitations to our study despite the novel findings from the scale development process. First, the findings from our study may be challenged because 2 of the experts from the round 1 evaluation did not return the round 2 evaluation forms. This may have affected the degree of agreement from round 1 to round 2 . The review panel in round 2 still included experts with similar expertise comparable with the panel in the round 1, which minimized the effect of losing feedback from 2 experts. Second, the initial plan was to meet each expert to explain the goal and the content evaluation steps, meeting all the reviewers was not achievable because of geographic and time barriers. 


\section{Conclusions}

This study provided evidence of content and face validity of the Tele-Pulmonary Rehabilitation Acceptance Scale. The current study developed 2 versions. One version with 13 items to measure telerehabilitation acceptance among patients with chronic respiratory diseases and one version with 17 items to measure telerehabilitation acceptance among health-care practitioners who worked in pulmonary rehabilitation programs. The Tele-Pulmonary Rehabilitation Acceptance Scale has demonstrated evidence of content validity as evaluated by a panel of experts in the fields of pulmonary rehabilitation, telehealth, information technology, and scale development. The Tele-Pulmonary Rehabilitation Acceptance Scale is significant because it provides one standardized data collection tool to measure telerehabilitation acceptance among potential users. Because using telerehabilitation is still a new field of practice, measuring its acceptance is an essential step before starting the clinical applications. Measuring acceptance of patients and health-care practitioners will help to assure successful implementation and positive outcomes of future telerehabilitation programs.

\section{REFERENCES}

1. Epping-Jordan J, World Health Organization. Innovative care for chronic conditions: building blocks for action: global report. Geneva, Switzerland: Noncommunicable Diseases and Mental Health, World Health Organization; 2002.

2. Hodgkin JE, Celli BR, Connors GL. Pulmonary Rehabilitation: Guidelines to Success. Mosby/Elsevier; 2009.

3. Spruit MA, Singh SJ, Garvey C, ZuWallack R, Nici L, Rochester C, et al; ATS/ERS Task Force on Pulmonary Rehabilitation. An official American Thoracic Society/European Respiratory Society statement: key concepts and advances in pulmonary rehabilitation. Am J Respir Crit Care Med 2013;188(8):e13-e64.

4. Wade VA, Eliott JA, Hiller JE. Clinician acceptance is the key factor for sustainable telehealth services. Qual Health Res 2014;24(5):682-694.

5. Alsubaiei ME, Cafarella PA, Frith PA, McEvoy RD, Effing TW. Barriers for setting up a pulmonary rehabilitation program in the Eastern Province of Saudi Arabia. Ann Thorac Med 2016;11(2):121-127.

6. Liu XL, Tan JY, Wang T, Zhang Q, Zhang M, Yao LQ, Chen JX. Effectiveness of home-based pulmonary rehabilitation for patients with chronic obstructive pulmonary disease: a meta-analysis of randomized controlled trials. Rehabil Nurs 2014;39(1):36-59.
7. Keating A, Lee A, Holland AE. What prevents people with chronic obstructive pulmonary disease from attending pulmonary rehabilitation? A systematic review. Chron Respir Dis 2011;8(2):89-99.

8. Brewster L, Mountain G, Wessels B, Kelly C, Hawley M. Factors affecting front line staff acceptance of telehealth technologies: a mixed-method systematic review. J Adv Nurs 2014;70(1):21-33.

9. Almojaibel A. Delivering pulmonary rehabilitation for patients with chronic obstructive pulmonary disease at home using telehealth: A review of the literature. Saudi J Med Medical Sci 2016;4(3):164-171.

10. Huis in 't Veld RMHA, Kosterink SM, Barbe T, Lindegård A, Marecek T, Vollenbroek-Hutten MM. Relation between patient satisfaction, compliance and the clinical benefit of a teletreatment application for chronic pain. J Telemed Telecare 2010;16(6):322-328.

11. Jayasuriya R, Caputi P. Computer attitude and computer anxiety in nursing. Validation of an instrument using an Australian sample. Nurses Computer Attitudes Inventory (NCATT) Computer Attitude Scale (CATT). Comput Nurs 1996;14(6):340-345.

12. Asaro PV, Williams J, Banet GA. Measuring the effect of a computerized nursing documentation system with objective measures and reported perceptions. Ann Emerg Med 2004;44(4):S131-S132.

13. Davis FD. Perceived usefulness, perceived ease of use, and user acceptance of information technology. MIS Q 1989;13(3):319-340.

14. Ma Q, Liu L. The technology acceptance model: a meta-analysis of empirical findings. J Organizational and End User Computing 2004; 16(1):59-72.

15. Rho MJ, Choi IY, Lee J. Predictive factors of telemedicine service acceptance and behavioral intention of physicians. Int J Med Inform 2014;83(8):559-571

16. Willard Van De B, Saovapa W. Exploring students' intention to use LINE for academic purposes based on technology acceptance model. Int Rev Res Open Distance Learning 2015;16(3):65-85.

17. Ashraf AR, Narongsak T, Seigyoung A. The application of the technology acceptance model under different cultural contexts: the case of online shopping adoption. J Int Marketing 2014;22(3):68-93.

18. Polit DF, Beck CT. The content validity index: are you sure you know what's being reported? Critique and recommendations. Res Nurs Health 2006;29(5):489-497.

19. Rogante M, Grigioni M, Cordella D, Giacomozzi C. Ten years of telerehabilitation: a literature overview of technologies and clinical applications. NeuroRehabilitation 2010;27(4):287-304.

20. Liu L, Miguel Cruz A, Rios Rincon A, Buttar V, Ranson Q, Goertzen D. What factors determine therapists' acceptance of new technologies for rehabilitation - a study using the Unified Theory of Acceptance and Use of Technology (UTAUT). Disabil Rehabil 2015;37(5): 447-455.

21. Seidman Z, McNamara R, Wootton S, Leung R, Spencer L, Dale M, et al. People attending pulmonary rehabilitation demonstrate a substantial engagement with technology and willingness to use telerehabilitation: a survey. J Physiother 2017;63(3):175-181.

This article is approved for Continuing Respiratory Care Education credit. For information and to obtain your CRCE

(free to AARC members) visit www.rcjournal.com

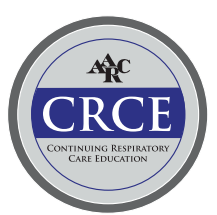

